\title{
(3200) Phaethon: Bulk density from Yarkovsky drift detection
}

\author{
J. Hanuš ${ }^{1}$, D. Vokrouhlický1 ${ }^{\text {, M. Delbo, }}{ }^{2}$, D. Farnocchia ${ }^{3}$, D. Polishook ${ }^{4}$, P. Pravec ${ }^{5}$, K. Hornoch ${ }^{5}$, H. Kučáková ${ }^{5}$, \\ P. Kušnirák ${ }^{5}$, R. Stephens ${ }^{6}$, and B. Warner ${ }^{7}$ \\ 1 Institute of Astronomy, Charles University, Prague, V Holešovičkách 2, 18000 Prague 8, Czech Republic \\ e-mail: hanus.home@gmail.com \\ 2 Université Côte d'Azur, Observatoire de la Côte d'Azur, CNRS, Laboratoire Lagrange, France \\ 3 Jet Propulsion Laboratory, California Institute of Technology, Pasadena, CA 91109, USA \\ ${ }^{4}$ Department of Earth and Planetary Sciences, Weizmann Institute of Science, Rehovot 0076100, Israel \\ 5 Astronomical Institute, Academy of Sciences of the Czech Republic, Fričova 1, 25165 Ondřejov, Czech Republic \\ ${ }^{6}$ Center for Solar System Studies, 11355 Mount Johnson Court, Rancho Cucamonga, CA 91737, USA \\ 7 Center for Solar System Studies, 446 Sycamore Ave., Eaton, CO 80615, USA
}

Received 12 September 2018 / Accepted 23 November 2018

\begin{abstract}
Context. The recent close approach of the near-Earth asteroid (3200) Phaethon offered a rare opportunity to obtain high-quality observational data of various types.

Aims. We used the newly obtained optical light curves to improve the spin and shape model of Phaethon and to determine its surface physical properties derived by thermophysical modeling. We also used the available astrometric observations of Phaethon, including those obtained by the Arecibo radar and the Gaia spacecraft, to constrain the secular drift of the orbital semimajor axis. This constraint allowed us to estimate the bulk density by assuming that the drift is dominated by the Yarkovsky effect.

Methods. We used the convex inversion model to derive the spin orientation and 3D shape model of Phaethon, and a detailed numerical approach for an accurate analysis of the Yarkovsky effect.

Results. We obtained a unique solution for Phaethon's pole orientation at $\left(318^{\circ},-47^{\circ}\right)$ ecliptic longitude and latitude (both with an uncertainty of $5^{\circ}$ ), and confirm the previously reported thermophysical properties $\left(D=5.1 \pm 0.2 \mathrm{~km}, \Gamma=600 \pm 200 \mathrm{~J} \mathrm{~m}^{-2} \mathrm{~s}^{-0.5} \mathrm{~K}^{-1}\right)$. Phaethon has a top-like shape with possible north-south asymmetry. The characteristic size of the regolith grains is $1-2 \mathrm{~cm}$. The orbit analysis reveals a secular drift of the semimajor axis of $-(6.9 \pm 1.9) \times 10^{-4} \mathrm{au} \mathrm{Myr}^{-1}$. With the derived volume-equivalent size of $5.1 \mathrm{~km}$, the bulk density is $1.67 \pm 0.47 \mathrm{~g} \mathrm{~cm}^{-3}$. If the size is slightly larger $\sim 5.7-5.8 \mathrm{~km}$, as suggested by radar data, the bulk density would decrease to $1.48 \pm 0.42 \mathrm{~g} \mathrm{~cm}^{-3}$. We further investigated the suggestion that Phaethon may be in a cluster with asteroids (155140) 2005 UD and (225416) 1999 YC that was formed by rotational fission of a critically spinning parent body.

Conclusions. Phaethon's bulk density is consistent with typical values for large $(>100 \mathrm{~km})$ C-complex asteroids and supports its association with asteroid (2) Pallas, as first suggested by dynamical modeling. These findings render a cometary origin unlikely for Phaethon.
\end{abstract}

Key words. minor planets, asteroids: individual: (3200) Phaethon - methods: numerical - methods: observational - astrometry celestial mechanics

\section{Introduction}

Physical properties of the low-perihelion near-Earth asteroid Phaethon, target of the proposed JAXA DESTINY ${ }^{+}$mission (Arai et al. 2018), have so far been inferred from a wide range of datasets: photometry in optical (Krugly et al. 2002; Ansdell et al. 2014) and infrared bands (Green et al. 1985; Tedesco et al. 2004), spectroscopy (Licandro et al. 2007; Hanuš et al. 2016), polarimetry (Devogèle et al. 2018; Ito et al. 2018), and radar (Taylor et al. 2018). Other studies were dedicated to Phaethon's comet-like activity (Jewitt \& Li 2010; Jewitt et al. 2013; Li \& Jewitt 2013; Ye et al. 2018), to the associated Geminid meteor stream (Gustafson 1989; Williams \& Wu 1993; Trigo-Rodríguez et al. 2004), or to the dynamical link with the main-belt asteroid (2) Pallas (de León et al. 2010; Todorović 2018).

Despite these numerous studies, the true nature of Phaethon has not yet been convincingly revealed. There is even a controversy involving the basic physical properties of Phaethon, such as its size and geometric visible albedo: recent studies based on polarimetric observations (Devogèle et al. 2018; Ito et al. 2018) report a significantly lower geometric albedo than has been inferred from thermal infrared data. Similarly, a possibly larger size than previous diameter determinations from thermal infrared observations was reported from the analysis of delay-Doppler observations by Taylor et al. (2018). These persisting inconsistencies motivated us to apply independent methods and improve our previous thermophysical modeling to ultimately understand the nature and origin of this intriguing object.

The polarimetric and dynamical studies mentioned above are in favor of a physical link between Phaethon and Pallas. In their view, Phaethon is an escapee member from the Pallas collisional family. This association is also supported by the spectroscopic studies in the visible and near-infrared (Licandro et al. 2007; de León et al. 2012). On the other hand, 
some authors remain in favor of a cometary origin for Phaethon (e.g., Trigo-Rodríguez et al. 2004; Borovička et al. 2010). In order to shed light on this fundamental issue, we first determine a unique model of Phaethon's spin state and its shape from a thermophysical model. Next, we use all available astrometric data to prove that accurate orbit determination requires that the Yarkovsky effect is included, which results in a steady decrease of the semimajor axis (e.g., Vokrouhlický et al. 2015). Because of its non-gravitational origin, a detailed theoretical model of the Yarkovsky effect, if fed by our spin and shape solution, allows us to constrain Phaethon's bulk density. This parameter helps us to infer its internal composition and conclude about its origin.

\section{Astrometric observations and orbit determination}

Astrometric data exist since the discovery of Phaethon by the Infrared Astronomical Satellite in October 1983 (Green \& Kowal 1983). As of July 2018, 4782 astrometric observations have been reported to the Minor Planet Center by ground-based observatories and 28 observations by the WISE spacecraft ${ }^{1}$. To this dataset of optical astrometry, we applied the Farnocchia et al. (2015a) star catalog debiasing and the Vereš et al. (2017) weighting scheme. Isolated observations that showed localized biases or internal inconsistencies were deweighted or excluded from the fit.

In addition to the ground-based observations and WISE data, the Gaia spacecraft observed Phaethon during 12 transits between September 2014 and February 2016. The corresponding astrometry was part of the Gaia DR2 release (Gaia Collaboration 2018). The Gaia small-body astrometry is decoupled into two components, along scan (AL), and across scan (AC). These two components are only weakly correlated; the AL component has a much greater accuracy than the AC component. Nevertheless, we made use of the full correlated observation error covariance model and found that the characteristic uncertainties were $\simeq 10$ mas in the $\mathrm{AL}$ direction and $\simeq 0.6^{\prime \prime}$ in the AC direction. To avoid problems with transit-specific systematic errors, we selected a single observation for each of the 12 transits.

Because of its low Earth MOID of $\simeq 0.02 \mathrm{au}$, Phaethon occasionally experiences close approaches to the Earth. During the 2007 and 2017 approaches, a total of six delay measurements were collected from the Arecibo and Goldstone radars (Taylor et al. 2018) ${ }^{2}$. This wealth of observational data places extremely tight constraints on the orbit of Phaethon. For instance, the formal uncertainty in semimajor axis is only $\simeq 64 \mathrm{~m}$ and only $20 \mathrm{~ms}$ in orbital period.

We used the aforementioned astrometric observations for orbital determination: Our force model includes the Newtonian gravity of the Sun, the planets, Pluto, Moon, the 16 largest perturbers in the main belt, and relativistic effects (e.g., Farnocchia et al. 2015b). Since Phaethon has a low perihelion and experiences close encounters to the Earth, we also included perturbations that are due the oblateness of the Sun and the Earth. To fit the complete dataset, and in particular both the 2007 and 2017 radar apparitions, it was also necessary to include nongravitational perturbations, in particular, the Yarkovsky effect. We describe Yarkovsky modeling and its parametric dependence in Sect. 3.4.

\footnotetext{
1 https://www.minorplanetcenter.net/db_search/show_ object?utf8=\%E2\%9C\%93\&object_id=3200

2 https://ssd.jpl . nasa.gov/?grp=ast\&fmt=html\&radar=
}

\section{Results}

\subsection{Revised spin and shape model}

We applied the convex inversion method of Kaasalainen et al. (2001) and Kaasalainen \& Torppa (2001) to the optical dataset of 70 light curves (described in Appendix A and listed in Table A.1) following exactly the procedure of Hanuš et al. (2016). Specifically, we scanned rotation periods in the proximity of the expected value while testing ten initial pole solutions for each sampled period. Four poles were selected on the equator with $90^{\circ}$ difference in longitude, and three poles in each hemisphere with the latitude $\pm 60^{\circ}$ and with $120^{\circ}$ difference in longitude. We assumed that all solutions within a $3 \sigma$ uncertainty interval had $\chi^{2}<(1+3 \sqrt{2 / v}) \chi_{\min }^{2}$, where $\chi_{\min }^{2}$ is the $\chi^{2}$ of the bestfitting solution and $v$ is the number of degrees of freedom. This threshold to consider the solution acceptable was used before in Vokrouhlický et al. (2017) or Durech et al. (2018) ${ }^{3}$ and corresponded to a $\sim 7 \%$ increase in $\chi_{\min }^{2}$ value. Only the best-fitting solution fulfilled the $3 \sigma$ condition on the $\chi^{2}$. To further verify that the best-fitting solution was the only one acceptable, we also visually inspected the light-curve fit with the second-best-fitting period, similarly as in Hanuš et al. (2016), see their Figs. 3 and 4 for illustration. This solution was already inconsistent with several individual light-curves. Therefore, we considered the difference in $\chi^{2}$ as significant and rejected all periods except for the best-fitting one. Next, we ran the convex inversion with the unique period and multiple pole orientations (isotropically distributed on a sphere with a $30^{\circ}$ difference) as starting points of the optimization procedure and derived a single solution within the $3 \sigma$ uncertainty interval defined above. Again, we visually inspected the light-curve fit with the second-best-fitting pole orientation and rejected this solution and also considered all other solutions as non-acceptable. The final solution is given in Table 1 together with the previous determinations. It is notable that our analysis and the recent study of Kim et al. (2018) provide for the first time a unique shape and spin solution that is consistent with the preferred solution of Hanuš et al. (2016). There are two differences between our old and revised models: (i) the relative dimension along the rotation axis (or the $c / a$ ratio) is now smaller by $\sim 10 \%$, which is expected because this dimension is generally the least constrained by the optical data, and (ii) the pole directions are about $8^{\circ}$ apart.

We find that the overall shape of Phaethon is nearly axially symmetric: the $x-y$ projection is not far from a circle with $b / a$ of $\sim 0.94$ (right panel of Fig. 1). Moreover, there seems to be a hint of an equatorial ridge and the top-shape like appearance (also noted by Taylor et al. 2018, from radar observations) that is often found in the sub-kilometer and kilometer-sized fast rotators (Ostro et al. 2006; Busch et al. 2011; Naidu et al. 2015). Additionally, our model suggests a north-south asymmetry of Phaethon, with the northern hemisphere slightly suppressed. Interestingly, our model for Phaethon is reminiscent of that of the Hayabusa 2 mission target (162173) Ryugu, whose recent public images revealed its top-shape appearance (Hasegawa et al. 2008).

\subsection{Updated thermophysical properties}

Given the new and unique spin and shape model, we repeated the thermophysical modeling (TPM) of Hanuš et al. (2016). The revised TPM solution is consistent with the previous one, mostly because the shape model derived here is similar to that

\footnotetext{
3 Be aware of the typo in the referenced equation in these two studies.
} 
Table 1. Rotation state parameters derived for Phaethon from different photometric datasets.

\begin{tabular}{|c|c|c|c|c|c|}
\hline $\begin{array}{c}\lambda_{1} \\
(\mathrm{deg})\end{array}$ & $\begin{array}{c}\beta_{1} \\
(\mathrm{deg})\end{array}$ & $\begin{array}{c}\lambda_{2} \\
(\mathrm{deg})\end{array}$ & $\begin{array}{c}\beta_{2} \\
(\mathrm{deg})\end{array}$ & $\begin{array}{c}P \\
(\mathrm{~h}) \\
\end{array}$ & Reference \\
\hline $276 \pm 15$ & $-15 \pm 15$ & $\begin{array}{l}97 \pm 15 \\
85 \pm 13\end{array}$ & $\begin{array}{l}-11 \pm 15 \\
-20 \pm 10\end{array}$ & $\begin{array}{ll}3.5906 & \pm 0.0001 \\
3.6032 & \pm 0.0008\end{array}$ & $\begin{array}{l}\text { Krugly et al. (2002) } \\
\text { Ansdell et al. (2014) }\end{array}$ \\
\hline $\begin{array}{l}319 \pm 5 \\
308 \pm 10 \\
318 \pm 5\end{array}$ & $\begin{array}{l}-39 \pm 5 \\
-52 \pm 10 \\
-47 \pm 5\end{array}$ & $84 \pm 5$ & $-39 \pm 5$ & $\begin{array}{l}3.603958 \pm 0.000002 \\
3.603957 \pm 0.000001 \\
3.603957 \pm 0.000001\end{array}$ & $\begin{array}{c}\text { Hanuš et al. (2016) } \\
\text { Kim et al. (2018) } \\
\text { This work }\end{array}$ \\
\hline
\end{tabular}

Notes. The table gives the ecliptic longitude $\lambda$ and latitude $\beta$ of all possible pole solutions, the sidereal rotation period $P$, and the reference.

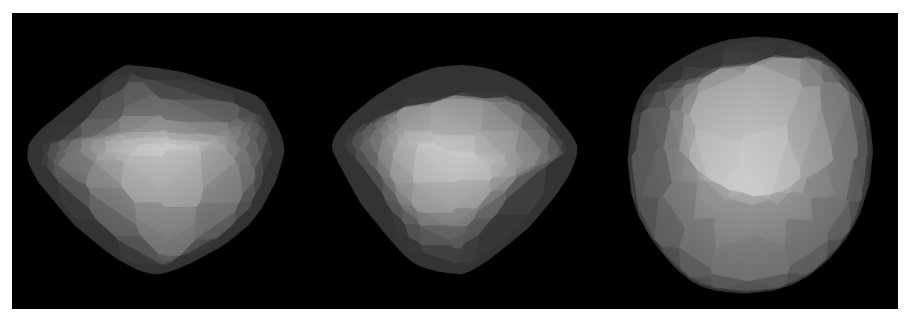

Fig. 1. Revised shape model of Phaethon. Three different viewing geometries are shown: the left and middle images are equator-on views rotated by $90^{\circ}$, the right image is a pole-on view.

of Hanuš et al. (2016). Therefore, we do not report in detail the new results as they are essentially identical to those in Hanuš et al. (2016): (i) equivalent size $D=5.1 \pm 0.2 \mathrm{~km}$, (ii) geometric albedo $p_{\mathrm{V}}=0.122 \pm 0.008$, and (iii) thermal inertia $\Gamma=600 \pm 200 \mathrm{~J} \mathrm{~m}^{-2} \mathrm{~s}^{-0.5} \mathrm{~K}^{-1}$ (all formal uncertainties).

\subsection{Regolith grain size and thermal conductivity}

We used the method of Gundlach \& Blum (2013) to determine the grain size of the surface regolith of Phaethon. The method uses the asteroid thermal inertia $\Gamma=600 \pm 200 \mathrm{~J} \mathrm{~m}^{-2} \mathrm{~s}^{-0.5} \mathrm{~K}^{-1}$ that was obtained by Hanuš et al. (2016) has also been confirmed by us here to infer the thermal conductivity $\kappa$ of the regolith,

$\kappa=\frac{\Gamma^{2}}{\phi \rho c}$,

where $c$ is the specific heat capacity, and $\rho$ is the grain density, as a function of the regolith grain filling factor $\phi$. These conductivity values are represented by the horizontal lines in Fig. 2.

Since the regolith filling factor is unconstrained, we considered its values to range between 0.1 (extremely fluffy packing, which is plausible only for small regolith particles) and 0.6 (close to the densest packing of equal-sized particles) with $\Delta \phi=0.1$ step. The values of $\rho$ and $c$ represent typical C-type thermophysical properties of the regolith (see Opeil et al. 2010). We only used the nominal $\Gamma$ value for the grain size determination. The relative uncertainty of $30 \%$ in the value of the thermal inertia translates into variations of the horizontal lines in Fig. 2 of about $60-70 \%$, which is still within the range encompassed by our lack of knowledge of the value of $\phi$.

Next, the Gundlach \& Blum (2013) model calculates the thermal conductivity of a granular medium (the regolith) as a function of the size of the grain and temperature, assuming density, heat capacity, and thermal conductivity of the solid grains. The thermal inertia for Phaethon was derived from observations obtained at $1.1 \mathrm{au}$ and $1 \mathrm{au}$ from the Sun. Using $p_{\mathrm{V}}=0.122$ and $G=0.15$ from Hanuš et al. (2016), we obtained a value of

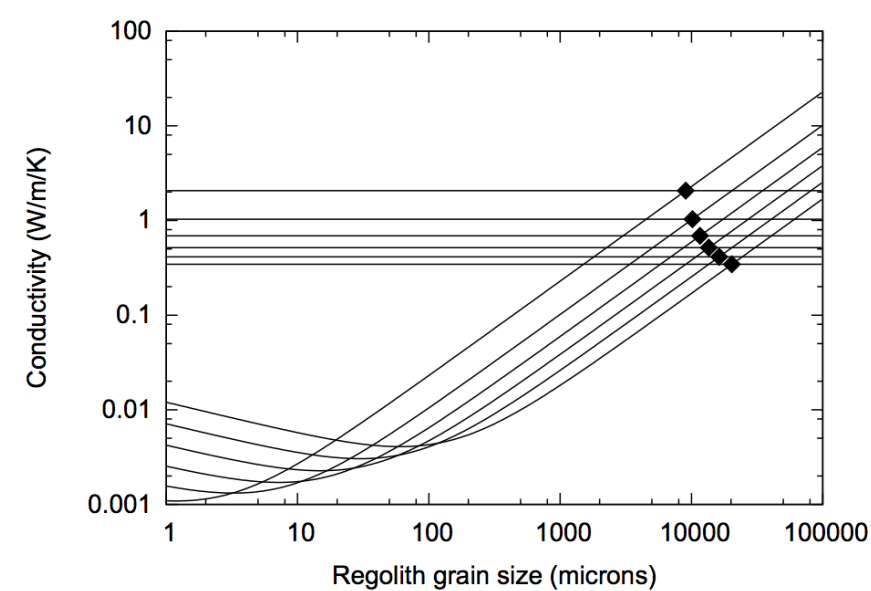

Fig. 2. Phaethon's regolith grain-size. Horizontal lines indicate the derived values of the thermal conductivity, following Eq. (1), for the different volume filling factors $\phi$ of the material and for the nominal thermal inertia value of $600 \mathrm{~J} \mathrm{~m}^{-2} \mathrm{~s}^{-0.5} \mathrm{~K}^{-1}$ (from top to bottom: $\phi=0.6,0.5,0.4,0.3,0.2$, and 0.1$)$. The curves represent the thermal conductivity of a regolith with average thermophysical properties of CC meteorites (see Opeil et al. 2010) as a function of the regolith grain size again for the different volume filling factors $\phi$. The intersection of the curves with the horizontal lines (for the same $\phi$ ) give the inferred characteristic grain size of the regolith at the abscissa.

the bolometric Bond albedo $A=0.048$. Using $\eta=1.6$ as in Harris (1998), we estimate sub-solar temperature $T_{\mathrm{SS}}$ of $339 \mathrm{~K}$ and $355 \mathrm{~K}$ at $1.1 \mathrm{au}$ and $1 \mathrm{au}$, respectively. We used the mean of these values, namely $347 \mathrm{~K}$. Using these input parameters for the model, we now determined the curves in Fig. 2 following the model of Gundlach \& Blum (2013). The abscissa value of the intersection points between these curves and the horizontal lines of Fig. 2 indicate the typical radius of the regolith grains. We find that the typical regolith grain size for Phaethon is about $1-2 \mathrm{~cm}$.

The regolith volume filling factors of 0.1 or 0.2 are probably unrealistic for the larger grains derived above, so we decided to consider only the four cases listed in Table 2. Using Eq. (5) of Gundlach \& Blum (2013), we calculated the temperature-dependent thermal conductivity $\kappa$ for these four cases for temperatures between 100 and $1300 \mathrm{~K}$. For the temperature range and regolith grain size appropriate for Phaethon, Eq. (5) of Gundlach \& Blum (2013) gives $\kappa \propto T^{3}$ and may be approximated with a simpler relation in the form (Keihm 1984; Delbo' et al. 2015)

$\kappa=\kappa_{\mathrm{b}}+a T^{3}$

where the first term corresponds to the solid-state thermal conductivity $\kappa_{\mathrm{b}}$, the second term is due to thermal conductivity by 
Table 2. Four cases of the regolith volume filling factor $\phi$ and regolith grain size $r_{\mathrm{g}}$ combinations, and the corresponding parameters of the thermal conductivity dependence on the temperature $T$ given by Eq. (2).

\begin{tabular}{ccccc}
\hline \hline Case & $\phi$ & $\begin{array}{c}r_{\mathrm{g}} \\
(\mathrm{mm})\end{array}$ & $\begin{array}{c}\kappa_{\mathrm{b}}\left(\times 10^{-5}\right) \\
\left(\mathrm{W} \mathrm{m}^{-1} \mathrm{~K}^{-1}\right)\end{array}$ & $\begin{array}{c}a\left(\times 10^{-8}\right) \\
\left(\mathrm{W} \mathrm{m}^{-1} \mathrm{~K}^{-4}\right)\end{array}$ \\
\hline 1 & 0.3 & 12 & $8.8 \pm 1.4$ & 1.7 \\
2 & 0.4 & 14 & $11 \pm 1$ & 1.3 \\
3 & 0.5 & 16 & $20 \pm 2$ & 0.97 \\
4 & 0.6 & 20 & $32 \pm 3$ & 0.81 \\
\hline
\end{tabular}

photons, and $T$ is the temperature. By fitting Eq. (2) to the calculated values, we obtained the parameter values reported in Table 2.

\subsection{Yarkovsky drift detection and bulk density}

Because of its extremely eccentric orbit $(e \simeq 0.9)$, Phaethon presents a particularly challenging case for an accurate analysis of the Yarkovsky effect. As a result of the temperaturedependent thermal conductivity described by Eq. (2), the value of this parameter changes by a factor $\simeq 20$ between perihelion (0.14 au) and 1 au. Similarly, the thermal parameter, directly dictating angular lag of the thermal effects (e.g., Bottke et al. 2006), may change by a factor of $\simeq 5$ between perihelion and $1 \mathrm{au}$, and the radiation flux changes by a factor of $\simeq 50$ in the same range. All these large variations limit the validity of simplified approaches to the Yarkovsky effect modeling, and warrant the adoption of a fully fledged numerical approach.

We adopted the model developed by Čapek et al. (2005), in which 1D thermal conduction below each of the surface facets is solved numerically with the nonlinear Robin boundary condition at the surface, and the assumption of an isothermal core at a sufficient depth is made. A temperature-dependence of the thermal conductivity following Eq. (2) was used. For the sake of simplicity, the specific heat capacity $c$ was assumed constant, $c=560 \mathrm{~J} \mathrm{~kg}^{-1} \mathrm{~K}^{-1}$, and the regolith grain density obtained for C-type meteorites was used, $\rho=3.11 \mathrm{~g} \mathrm{~cm}^{-3}$ (both from Gundlach \& Blum 2013). We ran solutions for four values of the packing factor $\phi$ in the range between 0.3 and 0.6. Each time, the parameters of the thermal conductivity were adjusted to satisfy the constraints from thermal observations described in Sect. 3.3 (see Table 2). The time domain of one revolution about the Sun was divided into steps of $60 \mathrm{~s}$, short enough when compared to the $\simeq 3.6 \mathrm{~h}$ rotation period, and the space grid describing the depth below each of the surface increased exponentially, as described in Čapek et al. (2005). We ensured that at each depth, the von Neumann stability condition was satisfied. Typically, ten iterative steps of the algorithm provide the temperature with an accuracy of one degree or better in the whole space and time domain of the solution. The shape and spin state of Phaethon was taken from the modeling in Sect. 3.1. Similarly, the volume-equivalent size of $5.1 \mathrm{~km}$ from Sect. 3.2 was used as an implicit value. The last parameter required to compute the thermal recoil acceleration (the Yarkovsky effect) is the bulk density of Phaethon. Our nominal models use $1 \mathrm{~g} \mathrm{~cm}^{-3}$ for the clarity, but we treated this value as a free parameter in the orbit determination process (similarly to what was done for asteroid Bennu in Chesley et al. 2014). Scaling to different densities is easily implemented by using the inverse-proportional dependence of the thermal acceleration on the bulk density. In our analysis we neglected the enhancement of the Yarkovsky effect that is due to surface roughness (Rozitis \& Green 2012). This effect could cause an increase in our bulk density estimate of less than $10 \%$, which is well within the formal uncertainty.

After determining the temperature of each surface facet, we evaluated the total thermal acceleration at every minute throughout the orbit using a numerical surface integration. For simplicity, we assumed the Lambert thermal emission law (e.g., Eq. (3) in Bottke et al. 2006). These thermal acceleration values were then used as part of the fit to the astrometry.

With this procedure, we estimated Phaethon's bulk density as $1.67 \pm 0.47 \mathrm{~g} \mathrm{~cm}^{-3}$. Variations due to the different packing factors are negligible $\left(<0.02 \mathrm{~g} \mathrm{~cm}^{-3}\right)$. Therefore, the differences stemming from a choice of the thermal model are much smaller than the formal uncertainty of the density solution from the fit to the astrometry. We also verified that calibrating the $\kappa(T)$ constants $\kappa_{\mathrm{b}}$ and $a$ to the value of the thermal inertia $\Gamma=400$ and $800 \mathrm{~J} \mathrm{~m}^{-2} \mathrm{~s}^{-0.5} \mathrm{~K}^{-1}$, that is, at one standard deviation from the nominal value, produces an insignificant variation in the bulk density solution ( $10 \%$ vs. the $28 \%$ formal uncertainty). Similarly, our solution assumes the nominal value of Phaethon's size, specifically $5.1 \mathrm{~km}$, which has only small fractional (formal) uncertainty of $\simeq 4 \%$. This may again be neglected with respect to the $28 \%$ fractional uncertainty of the density solution. If required, we may also express the Yarkovsky detection for Phaethon in the usual way as a $-(6.9 \pm 1.9) \times 10^{-4} \mathrm{au} \mathrm{Myr}^{-1}$ secular drift of the orbital semimajor axis (compare with data, e.g., in Vokrouhlický et al. 2015).

\section{Discussion}

The size and albedo solution from the thermal modeling may have a systematic error due to the possibly improper modeling of physical effects on the surface of this extreme body. This point of view is advocated by results of the recent radar campaign (e.g., Taylor et al. 2018) and extrapolation of the polarimetric measurements at large phase angles (Ito et al. 2018; Devogèle et al. 2018 ), both of which yield asomewhat larger size $\simeq(5.7-5.8) \mathrm{km}$ than the $5.1 \mathrm{~km}$ nominal value used in this work. Without a detailed understanding of this difference, we are not fully capable of correcting our basic thermal model to accommodate this difference. If we were to assume this larger size, we would obtain a bulk density of $\sim 1.48 \mathrm{~g} \mathrm{~cm}^{-3}$, which is within the estimate uncertainty. We only note that the bulk density would approach the $\simeq 1.27 \mathrm{~g} \mathrm{~cm}^{-3}$ value obtained for asteroid (101955) Bennu (Chesley et al. 2014), which is also a small B-type body. While this consistency is encouraging, it should not be overstated: we note, for instance, that the suggested source region for Bennu (Eulalia or new Polana asteroid families, Walsh et al. 2013; Bottke et al. 2015) is different from that of Phaethon and the spectra of these two bodies are somewhat different in the nearinfrared range (Campins et al. 2010).

Our density solution for Phaethon from the Yarkovsky model in Sect. 3.4 assumed a particular model of the temperature dependence of the surface thermal conductivity. While supported by theoretical arguments and measurements for the lunar regolith (e.g., Keihm 1984), we do not have a direct observational confirmation of this effect on Phaethon. On the other hand, Rozitis et al. (2018) measured a different dependence of the thermal inertia on temperature on (1036) Ganymed, (276049) 2002 $\mathrm{CE}_{26}$, and (1580) Betulia. This is because the calibration of Phaethon's surface conductivity derives from observations taken at a very restricted range of heliocentric distances. Moreover, we note that the bulk density solution depends on the conductivity 
assumption. For instance, if the conductivity were assumed constant (and not increasing toward smaller heliocentric distances), the bulk density would decrease to $1.08 \pm 0.30 \mathrm{~g} \mathrm{~cm}^{-3}$. In general, any shallower dependence of the surface thermal conductivity on heliocentric distance than that of our nominal model would imply a lower bulk density.

We assumed that the Yarkovsky effect is the dominant nongravitational effect in the orbital solution of Phaethon. While the effects of the Poynting-Robertson or solar wind drag are about two orders of magnitude smaller and may be safely neglected, some concern remains about influence of the mass loss near perihelion passages. We note that direct observational evidence is quite limited and reveals only short episodes of very weak activity (e.g., Li \& Jewitt 2013). Additionally, the assumed small size of the particles triggering the observed effects results in a quite low mass-loss rate (e.g., Ye et al. 2018). We estimated the corresponding dynamical effects in Appendix B and found that the resulting change in semimajor axis of Phaethon is at most an order of magnitude smaller than the Yarkovsky effect.

Additionally, we note that the spin state solution from Sect. 3.1 implies that Phaethon's pole regions are never irradiated from low zenith angles near perihelion passages. For instance, the south rotation pole is shadowed before the perihelion passage and becomes illuminated during and after the passage. However, the maximum solar elevation about the local horizon at the south pole is only about $25^{\circ}$ and quickly becomes even smaller within a week. The situation is opposite for the north rotation pole. As a result, we do not expect a huge increase in activity at the pole regions caused by the changing geometry of illumination near perihelion.

(3200) Phaethon appears to be in a cluster with asteroids (155140) 2005 UD and (225416) 1999 YC (Ohtsuka et al. 2006, 2008; Hanuš et al. 2016) ${ }^{4}$. The cluster 3200-155140-225416 could be formed by rotational fission of a critically spinning parent body (Scheeres 2007; Pravec et al. 2018, and references therein). To examine this hypothesis, we estimated the total secondary-to-primary mass ratio of the cluster $q$ from the absolute magnitudes of its three members, $H_{1}=14.31, H_{2}=17.2$, and $H_{3}=17.3$ (Hanuš et al. 2016, and MPC) using Eqs. (3) and (4) of Pravec et al. (2018): $q=0.034$. With the primary rotation period $P_{1}=3.60 \mathrm{~h}$, this agrees excellently well with the theory of cluster formation by rotational fission. Specifically, it falls very close to the nominal $P_{1}-q$ curve in Fig. 14 of Pravec et al. (2018), which nominally predicts $P_{1}=3.51 \mathrm{~h}$ for $q=0.034$, see Fig. C.1. In other words, the current rotation of Phaethon was slowed down from the original critical spin frequency by the formation and ejection of the two secondaries, with part of its original rotation energy and angular moment carried away by the escaping secondaries. The apparent top-like shape of Phaethon may be a product of the spin fission process, as observed for a number of primaries of near-Earth binary asteroids. We further note that this hypothesis should not be overestimated: the uncertainty about whether the three bodies are indeed dynamically related is still great. Further physical characterization of the two smaller bodies is required to better understand the properties of the suggested cluster.

Our derived Phaethon bulk density is consistent with values typical for large $(>100 \mathrm{~km}) \mathrm{C}$-complex asteroids (Carry 2012; Marchis et al. 2008; Hanuš et al. 2017). However, simi-

\footnotetext{
4 The $6 \mathrm{~m}$ diameter asteroid $2012 \mathrm{KT}_{42}$ may be also a member of the Phaethon cluster. Polishook et al. (2012) found it to be a B type, very similar to Phaethon.
}

larly sized C-complex asteroids should have higher porosity and therefore a slightly lower density than found here for Phaethon. We may only speculate that the extreme solar irradiation is capable of decreasing the macroporosity. Interestingly, the bulk density of Pallas is higher than the typical values for the $D>$ $100 \mathrm{~km}$ C-complex asteroids, so that the possible dynamical link with Phaethon (de León et al. 2010; Todorović 2018) is consistent with Phaethon's higher bulk density. Conversely, typical comets are found to have bulk densities far lower (often lower than $1 \mathrm{~g} \mathrm{~cm}^{-3}$, e.g., Weissman \& Lowry 2008). Our results thus speak against Phaethon being a comet in its nearly dormant phase.

Acknowledgements. This work has been supported by the Czech Science Foundation (JH grant $18-04514 \mathrm{~J}$, DV and the Ondřejov group grant 1700774S). Additional support from the Charles University Research program No. UNCE/SCI/023 is also acknowledged. MD acknowledges support from the French Centre National d'Études Spatiales (CNES). DP acknowledges the Koshland Foundation's support. Part of the research was carried out at the Jet Propulsion Laboratory, California Institute of Technology, under a contract with the National Aeronautics and Space Administration. We thank J. Giorgini for providing useful information about the radar astrometry of Phaethon.

\section{References}

Ansdell, M., Meech, K. J., Hainaut, O., et al. 2014, ApJ, 793, 50

Arai, T., Kobayashi, M., Ishibashi, K., et al. 2018, Lun. Planet. Sci. Conf., 49, 2570

Borovička, J. 2010, in Proceedings of the International Meteor Conference, 26th IMC, Bareges, France, 2007, eds. J. Rendtel, \& J. Vaubaillon, 42

Bottke, J. W. F., Vokrouhlický, D., Rubincam, D. P., \& Nesvorný, D. 2006, Annu. Rev. Earth Planet. Sci., 34, 157

Bottke, W. F., Vokrouhlický, D., Walsh, K. J., et al. 2015, Icarus, 247, 191

Busch, M. W., Ostro, S. J., Benner, L. A. M., et al. 2011, Icarus, 212, 649

Campins, H., Morbidelli, A., Tsiganis, K., et al. 2010, ApJ, 721, L53

Ďapek, D., \& Vokrouhlický, D. 2005, Dynamics of Populations of Planetary Systems, eds. Z. Knežević, \& A. Milani (Cambridge: Cambridge University Press)

Carry, B. 2012, Planet. Space Sci., 73, 98

Chesley, S. R., Farnocchia, D., Nolan, M. C., et al. 2014, Icarus, 235, 5

de León, J., Campins, H., Tsiganis, K., Morbidelli, A., \& Licandro, J. 2010, A\&A, 513, A26

de León, J., Pinilla-Alonso, N., Campins, H., Licandro, J., \& Marzo, G. A. 2012, Icarus, 218, 196

Delbo', M., Mueller, M., Emery, J., Rozitis, B., \& Capria, M. T. 2015, in Asteroids IV, eds. P. Michel, F. E. DeMeo, \& W. F. Bottke (Tucson: The University of Arizona Press), 107

Devogèle, M., Cellino, A., \& Borisov, G. 2018, MNRAS, 479, 3498

Durech, J., Sidorin, V., \& Kaasalainen, M. 2010, A\&A, 513, A46

Durech, J., Vokrouhlický, D., Pravec, P., et al. 2018, A\&A, 609, A86

Farnocchia, D., Chesley, S. R., Chamberlin, A. B., \& Tholen, D. J. 2015a, Icarus, 245, 94

Farnocchia, D., Chesley, S. R., Milani, A., Gronchi, G. F., \& Chodas, P. W. 2015b, in Asteroids IV, eds. P. Michel, F. E. DeMeo, \& W. F. Bottke (Tucson: The University of Arizona Press), 815

Gaia Collaboration (Spoto, F., et al.) 2018, A\&A, 616, A13

Green, S., \& Kowal, C. 1983, IAU, Circ, 3878

Green, S. F., Meadows, A. J., \& Davies, J. K. 1985, MNRAS, 214, 29P

Gundlach, B., \& Blum, J. 2013, Icarus, 223, 479

Gustafson, B. A. S. 1989, A\&A, 225, 533

Hanuš, J., Delbo', M., Vokrouhlický, D., et al. 2016, A\&A, 592, A34

Hanuš, J., Viikinkoski, M., Marchis, F., et al. 2017, A\&A, 601, A114

Harris, A. W. 1998, Icarus, 131, 291

Hasegawa, S., Müller, T. G., Kawakami, K., et al. 2008, PASJ, 60, S399

Hui, M.-T., \& Li, J. 2017, AJ, 153, 23

Ito, T., Ishiguro, M., Arai, T., et al. 2018, Nat. Commun., 9, 2486

Jakubík, M., \& Neslušan, L. 2015, MNRAS, 453, 1186

Jewitt, D., \& Li, J. 2010, AJ, 140, 1519

Jewitt, D., Li, J., \& Agarwal, J. 2013, ApJ, 771, L36

Kaasalainen, M., \& Torppa, J. 2001, Icarus, 153, 24

Kaasalainen, M., Torppa, J., \& Muinonen, K. 2001, Icarus, 153, 37

Keihm, S. J. 1984, Icarus, 60, 568

Kim, M.-J., Lee, H.-J., Lee, S.-M., et al. 2018, A\&A, 619, A123 
Krugly, Y. N., Belskaya, I. N., Shevchenko, V. G., et al. 2002, Icarus, 158, 294 Li, J., \& Jewitt, D. 2013, AJ, 145, 154

Licandro, J., Campins, H., Mothé-Diniz, T., Pinilla-Alonso, N., \& de León, J. 2007, A\&A, 461, 751

Marchis, F., Descamps, P., Baek, M., et al. 2008, Icarus, 196, 97

Naidu, S. P., Margot, J. L., Taylor, P. A., et al. 2015, AJ, 150, 54

Ohtsuka, K., Sekiguchi, T., Kinoshita, D., et al. 2006, A\&A, 450, L25

Ohtsuka, K., Arakida, H., Ito, T., Yoshikawa, M., \& Asher, D. J. 2008, Meteorit. Planet. Sci. Suppl., 43, 5055

Opeil, C. P., Consolmagno, G. J., \& Britt, D. T. 2010, Icarus, 208, 449

Ostro, S. J., Margot, J.-L., Benner, L. A. M., et al. 2006, Science, 314, 1276

Polishook, D., \& Brosch, N. 2009, Icarus, 199, 319

Polishook, D., Binzel, R. P., Lockhart, M., et al. 2012, Icarus, 221, 1187

Pravec, P., Fatka, P., Vokrouhlický, D., et al. 2018, Icarus, 304, 110

Rozitis, B., \& Green, S. F. 2012, MNRAS, 423, 367

Rozitis, B., Green, S. F., MacLennan, E., \& Emery, J. P. 2018, MNRAS, 477, 1782

Scheeres, D. J. 2007, Icarus, 189, 370
Taylor, P. A., Marshall, S. E., Venditti, F., et al. 2018, Lun. Planet. Sci. Conf., 49, 2509

Tedesco, E. F., Noah, P. V., Noah, M., \& Price, S. D. 2004, NASA Planetary Data System, 12

Todorović, N. 2018, MNRAS, 475, 601

Trigo-Rodríguez, J. M., Llorca, J., Borovička, J., \& Fabregat, J. 2004, Earth Moon and Planets, 95, 375

Vereš, P., Farnocchia, D., Chesley, S. R., \& Chamberlin, A. B. 2017, Icarus, 296, 139

Vokrouhlický, D., Bottke, W. F., Chesley, S. R., Scheeres, D. J., \& Statler, T. S. 2015, in The Yarkovsky and YORP, eds. P. Michel, F. E. DeMeo, \& W. F. Bottke, 509

Vokrouhlický, D., Pravec, P., Durech, J., et al. 2017, AJ, 153, 270

Walsh, K. J., Delbó, M., Bottke, W. F., Vokrouhlický, D., \& Lauretta, D. S. 2013, Icarus, 225, 283

Warner, B. D. 2017, Minor Planet Bull., 44, 98

Weissman, P. R., \& Lowry, S. C. 2008, Meteorit. Planet. Sci., 43, 1033

Williams, I. P., \& Wu, Z. 1993, MNRAS, 262, 231

Ye, Q., Wiegert, P. A., \& Hui, M.-T. 2018, ApJ, 864, L9 


\section{Appendix A: Optical light curves}

We downloaded 55 optical light curves from the DAMIT database (Ďrech et al. 2010) that have been already used for the shape model determination in Hanuš et al. (2016). Moreover, we enhanced this dataset by adding four light curves obtained in 2016 by Warner (2017), four light curves by David Polishook (apparitions in 2005, 2007 and 2017), and finally, four light curves obtained by Brian Warner, two by Robert Stephens and one by Petr Pravec during the most recent apparition in December 2017. We note that our new data sample three additional apparitions in 2005, 2016 and 2017. New observations are summarized in Table A.1. Additional details about the image/data processing can be found in Polishook \& Brosch (2009).

Table A.1. New optical photometry used to revise the shape model.

\begin{tabular}{|c|c|c|c|c|c|c|c|c|c|}
\hline $\mathrm{N}$ & Epoch & $N_{p}$ & $\begin{array}{c}\Delta \\
(\mathrm{au})\end{array}$ & $\begin{array}{c}r \\
(\mathrm{au}) \\
\end{array}$ & $\begin{array}{c}\varphi \\
(\mathrm{deg})\end{array}$ & Filter & Site & Observer & Reference \\
\hline 1 & $2005-11-27.0$ & 62 & 1.51 & 2.40 & 12.6 & $R$ & WISE & David Polishook & This work \\
\hline 2 & $2007-12-02.0$ & 93 & 0.21 & 1.07 & 62.2 & $R$ & WISE & David Polishook & This work \\
\hline 3 & $2016-11-02.2$ & 49 & 0.69 & 1.49 & 34.0 & $V$ & CS3-PDS & Brian Warner & Warner (2017) \\
\hline 4 & 2016-11-03.2 & 62 & 0.71 & 1.50 & 33.8 & $V$ & CS3-PDS & Brian Warner & Warner (2017) \\
\hline 5 & 2016-11-04.2 & 119 & 0.72 & 1.51 & 33.5 & $V$ & CS3-PDS & Brian Warner & Warner (2017) \\
\hline 6 & 2016-11-05.2 & 109 & 0.74 & 1.52 & 33.4 & $V$ & CS3-PDS & Brian Warner & Warner (2017) \\
\hline 7 & 2017-11-12.1 & 100 & 0.69 & 1.49 & 33.1 & $\mathrm{R}$ & WISE & David Polishook & This work \\
\hline 8 & $2017-11-23.2$ & 160 & 0.46 & 1.36 & 30.4 & $\mathrm{R}$ & D65 & $\begin{array}{r}\text { Petr Pravec, Hana Kučáková } \\
\text { Kamil Hornoch, Peter Kušnirák }\end{array}$ & This work \\
\hline 9 & $2017-11-26.3$ & 89 & 0.40 & 1.32 & 29.1 & $V$ & CS3-TRJ & Robert Stephens & This work \\
\hline 10 & $2017-12-01.3$ & 25 & 0.30 & 1.25 & 26.2 & $V$ & CS3-TRJ & Robert Stephens & This work \\
\hline 11 & 2017-12-01.5 & 24 & 0.30 & 1.25 & 26.1 & $V$ & CS3-PDS & Brian Warner & This work \\
\hline 12 & $2017-12-02.3$ & 21 & 0.28 & 1.23 & 25.5 & $V$ & CS3-PDS & Brian Warner & This work \\
\hline 13 & 2017-12-02.4 & 12 & 0.28 & 1.23 & 25.4 & $V$ & CS3-PDS & Brian Warner & This work \\
\hline 14 & $2017-12-02.5$ & 23 & 0.28 & 1.23 & 25.4 & $V$ & CS3-PDS & Brian Warner & This work \\
\hline 15 & 2017-12-09.0 & 58 & 0.16 & 1.14 & 19.7 & $R$ & WISE & David Polishook & This work \\
\hline
\end{tabular}

Notes. The table gives the epoch, the number of individual measurements $N_{\mathrm{p}}$, fighe asteroid distances to the Earth $\Delta$ and the Sun $r$, the phase angle $\varphi$, the photometric filter, and the observational log. WISE - Wise Observatory, Israel, CS3-PDS - Center for Solar System Studies, 446 Sycamore Ave., Eaton, CO 80615, USA, D65 - 65cm telescope at Ondřejov Observatory, Czech Republic, CS3-TRJ - Center for Solar System Studies, 11355 Mount Johnson Ct., Rancho Cucamonga, CA 91737, USA.

\footnotetext{
5 http://astro.troja.mff.cuni.cz/projects/asteroids3D
} 


\section{Appendix B: Acceleration due to mass loss}

Here we estimate the change in semimajor axis of Phaethon that is due to mass loss. The documented activity of Phaethon (e.g., Jewitt \& Li 2010; Jewitt et al. 2013; Li \& Jewitt 2013) is very tiny and restricted to a very narrow interval of time around perihelion passage. Jewitt et al. (2013) mention the 2009 and 2012 events as $\sim 2 \mathrm{~d}$ activity with an average mass loss rate $\mathrm{d} M / \mathrm{d} t \sim 3 \mathrm{~kg} \mathrm{~s}^{-1}$ and characteristic ejection speeds between $V \sim 10-30 \mathrm{~m} \mathrm{~s}^{-1}$ (the upper value being an order of magnitude higher than the escape speed from Phaethon, perhaps consistent with small size of the observed particles and radiative striping from the body, rather than a traditional jet-like activity). We note that the 2016 perihelion passage activity was even smaller (Hui \& Li 2017). With these numbers we obtain an estimate of the effective recoil acceleration

$a_{\mathrm{rec}} \sim V(\mathrm{~d} M / \mathrm{d} t) / M_{3200} \sim 6.4 \times 10^{-14} \mathrm{au} \mathrm{d}^{-2}$.

Here, $M_{3200}$ is the estimated mass of the body, conservatively assuming a smaller size of $5.1 \mathrm{~km}$ and only $1 \mathrm{~g} \mathrm{~cm}^{-3}$ bulk density.

We denote $T_{\text {act }}$ the time interval of activity around perihelion and assume $T_{\text {act }} \sim 5 \mathrm{~d}$, again longer than observed so far. For the sake of estimating the dynamical effect, we take now the most extreme possibility that all the observed particles are ejected in a narrow jet emanating from the north pole of Phaethon. Then the effective orbit-averaged change in semimajor axis is

$(\mathrm{d} a / \mathrm{d} t)_{\mathrm{eff}} \sim \frac{1}{\pi} \sqrt{((1+e) /(1-e))} T_{\mathrm{act}} a_{\mathrm{rec}} f$,

where the factor $\sqrt{((1+e) /(1-e))}$ stems from expressing the perihelion velocity and the coefficient $f$ is a projection factor of the recoil acceleration to the perihelion velocity vector direction. With our determined pole orientation we find $f \sim \cos (70 \mathrm{deg}) \sim$ $1 / 3$. By combining this, we obtain

$$
(\mathrm{d} a / \mathrm{d} t)_{\mathrm{eff}} \sim 0.54 \times 10^{-4} \mathrm{au} \mathrm{Myr}^{-1} .
$$

This is less then $10 \%$ of our found orbital decay of $-(6.9 \pm$ 1.9) $\times 10^{-4} \mathrm{au} \mathrm{Myr}^{-1}$.

We note that our assumptions were rather conservative. If the activity comes from lower latitudes at the body, another decrease of the effect should be expected. This is because the equatorial projection of the recoil will be averaged by fast rotation of the asteroid.

We admit that the observed activity is mainly in very small particles. There is no direct observational evidence of a possible ejection of larger particles during the recent perihelion passages. With this lack of observational constraints, it is hard to say anything about this component. It may appear that properties of the associated Geminid stream would be a guidance. We note, however, that studies of the Geminid activity (e.g., Jakubík \& Neslušan 2015), while indicating a rather young age not exceeding $1000 \mathrm{yr}$, cannot be directly taken as support for an equivalent activity within the last $30 \mathrm{yr}$ of the orbital data.

\section{Appendix C: Additional figure}

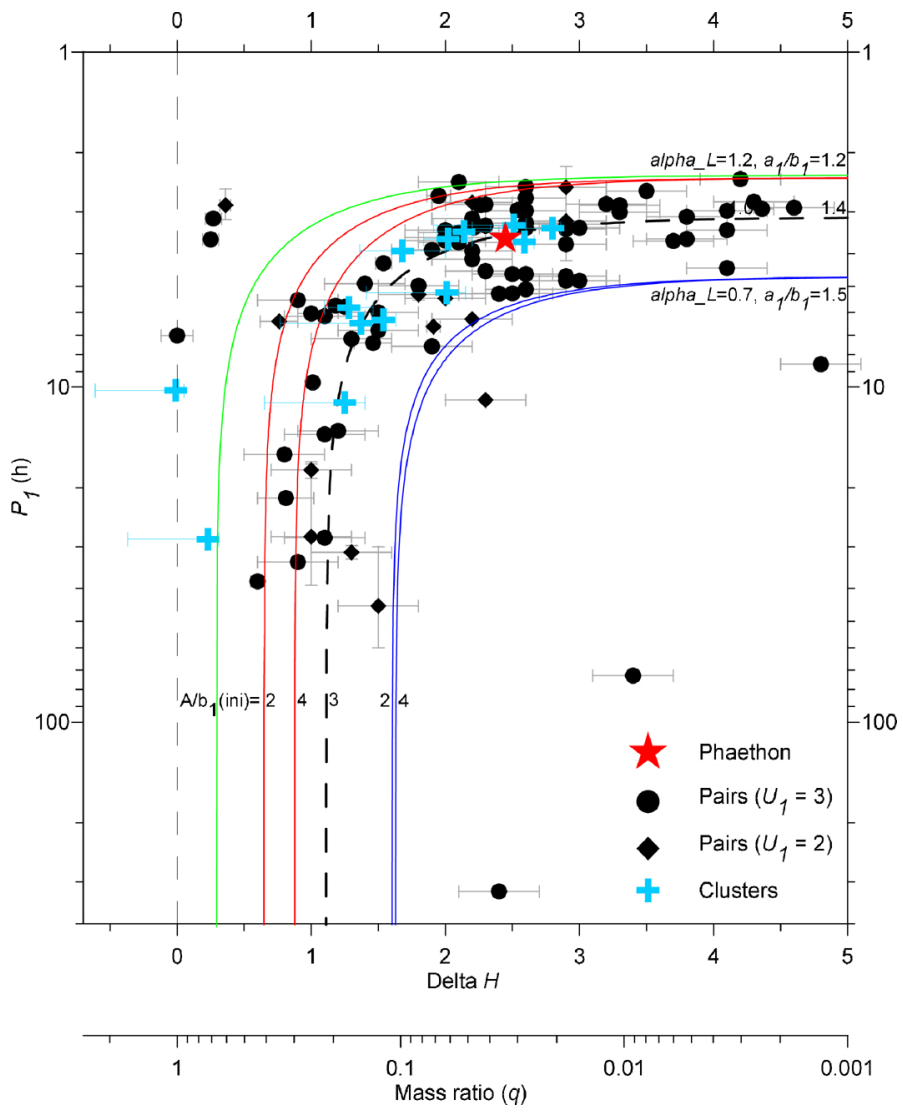

Fig. C.1. Distribution of primary rotation periods $P_{1}$ vs. the total secondary-to-primary mass ratios $q$ for 13 asteroid clusters, 93 asteroid pairs, and Phaethon. The figure is adopted from Pravec et al. (2018; Fig. 14), see that paper for a full description of the figure content. 